\begin{tabular}{||l|l|}
\hline Citation & $\begin{array}{l}\text { Nicolas Butzen, Michiel Steyaert, (2017), } \\
\text { Design of Soft-Charging Switched-Capacitor DC-DC Converters Using } \\
\text { Stage Outphasing and Multiphase Soft-Charging } \\
\text { Journal of Solid-State Circuits (JSSC), 52, pp. 3132-3141, Dec. 2017. }\end{array}$ \\
\hline Archived version & $\begin{array}{l}\text { Author manuscript: the content is identical to the content of the published } \\
\text { paper, but without the final typesetting by the publisher }\end{array}$ \\
\hline Published version & $\underline{\text { https://doi.org/10.1109/JSSC.2017.2733539 }}$ \\
\hline Journal homepage & http://sscs.ieee.org/publications/ieee-journal-of-solid-state-circuits-jssc \\
\hline Author contact & $\begin{array}{l}\text { nicolas.butzen@esat.kuleuven.be } \\
+32(0) 16325534\end{array}$ \\
\hline
\end{tabular}

(article begins on next page) 


\title{
Design of Soft-Charging Switched-Capacitor DC-DC Converters using Stage Outphasing and Multiphase Soft-Charging
}

\author{
Nicolas Butzen, Student Member, IEEE, and Michiel Steyaert, Fellow, IEEE
}

\begin{abstract}
In this paper, two techniques, called StageOutphasing (SO) and Multiphase Soft-Charging (MSC), are introduced, that make use of the advanced multiphasing concept to soft-charge charge transfers between flying capacitors. As such, the charge sharing losses of fully integrated switched-capacitor (SC) converters are reduced, leading to better capacitance utilization, higher efficiency and higher power-density. Furthermore, when used in combination with the Dickson converter, the relative improvement gets better with increasing voltage conversion ratio, making them an excellent choice to reduce power delivery network-induced losses in modern microchips. The impact of the proposed techniques on the performance are discussed. A 3:1 Dickson SC converter is realized that implements SO and MSC, and achieves a state-of-theart $1.1 \mathrm{~W} / \mathrm{mm}^{2}$ power-density, $82 \%$ efficiency combination using common capacitor technologies. Finally, two figure of merits for monolithic SC DC-DC converters are proposed that include the voltage conversion ratio and power density, to allow fair comparison of the converter's performance to literature.
\end{abstract}

Index Terms-Switched-Capacitor, DC-DC, Power Converter, Power Management, Soft-Charging, Adiabatic Charging, Power Density, Advanced Multiphasing

\section{INTRODUCTION}

$\mathbf{O}$ Ver the past years, there has been a steadilyincreasing interest in monolithic power conversion for a wide range of applications. On the low-power end, applications such as the internet-of-things (IoT) can benefit tremendously from the reduced size and billof-materials $(\mathrm{BoM})$ that an integrated power converter provides [1], [2], especially when combined with energy scavenging. For high-power digital loads, on the other hand, on-chip voltage conversion and regulation are becoming close to a necessity from an efficiency point of view. This is demonstrated by Fig. 1. With an external DC-DC converter the microchip requires a certain amount of intake current inversely proportional to the load's voltage. This intake current induces static- and dynamic voltage droops in the power delivery network

The authors are with the Department of Electrical Engineering, KU Leuven, B-3001 Heverlee, Belgium, e-mail: nicolas.butzen@esat.kuleuven.be .

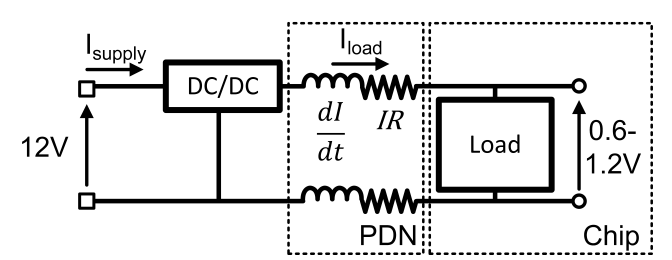

(a)

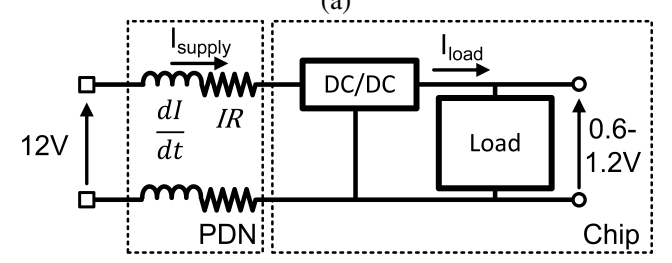

(b)

Fig. 1. Simplified schema of an example power delivery network (PDN) to an integrated load using (a) an external- and (b) an on-chip DC/DC converter.

(PDN), for which the digital load needs to implement a worst-case voltage guard-band to guarantee correct operation [3]. Because the load voltage of modern processor has continued to decrease over time [4], however, these effects have effectively become large loss contributors [5].

As an alternative, many have proposed shifting the DC-DC converter on-chip as shown in Fig. 1b [3], [5], [6]. This way, the intake current, together with current-induced droops, is ideally reduced by the onchip voltage conversion ratio (VCR) [7], the guard-bands can be minimized and the overall system efficiency is significantly increased.

In order to be viable, though, the fully integrated converter must satisfy a number of properties. Most importantly, it must have a high efficiency and VCR such that the losses it adds itself are smaller than the reduction of PDN losses it achieves. Generally speaking, the larger the VCR and the efficiency, the more suited the converter is for this situation. At the same time, the converter needs to maximize its power density to limit the area overhead in the system, and only require commonly-available devices to enable wide-spread use.

Switched-capacitor (SC) converters in particular have 
been shown to be an excellent candidate for monolithic power conversion [8] because they only require switches and capacitors, both of which are readily available in modern technology processes. That being said, the monolithic context does pose its own challenges and has a constraint design space, shown in Fig. 2. At low power densities, the converter's efficiency is limited by the ratio of the flying capacitor's parasitic substrate coupling, $C_{p a r}$ and the flying capacitance, $C_{f l y}$, itself due to a combination of charge-sharing- and bottomplate losses [9], and to a lesser extent the leakage-toconduction conduction ratio of the power transistors [10]. For increasingly large output powers, the power transistor losses gain in importance and a clear efficiencypower-density trade-off becomes apparent. Here, it is the ratio of the transistor $Q_{o n} R_{o n}$ and $C_{f l y}$ density that is the dominant factor [9]. Either way, the flying capacitance density plays a crucial role in both regimes. Furthermore, regardless of the power-density, the larger the VCR of a converter, the lower the efficiency that can be obtained.

Due to the inherent planar nature of modern technology nodes, the capacitance density is quite low and has been proven to be a severe burden, causing designs in literature to either achieve high efficiency at low power-density [11] $\left(94.6 \%\right.$ at $\left.1.3 \mathrm{~mW} / \mathrm{mm}^{2}\right)$, or low efficiency at high power-densities [12], [13] $(60 \%$ at $1.1 \mathrm{~W} / \mathrm{mm}^{2}, 0.77 \mathrm{~W} / \mathrm{mm}^{2}$ respectively). Converters using SOI or Deep-Trench capacitors have improved transistors or $>20 \mathrm{x}$ higher capacitance density at their disposal, causing them to perform significantly better as a result [9], [14], but are more expensive and/or rely on devices that are far from common. Moreover, all of these converters obtain their best result at a VCR of only $3: 2$ or $2: 1$, leaving much room to reduce PDN losses further.

Enabled by the low complexity cost and high frequency limit of modern technologies, combined with the low overhead cost of fragmentation, it is possible to improve the performance of SC converters by using multiple phase-shifted converter cores that actively interact with each other. The authors refer to this concept as Advanced Multiphasing (AM). In [10], [11], for example, such a technique was introduced that reduces the effective parasitic coupling in SC converters by softcharging them, thus increasing the efficiency ceiling at low power densities. A seperate, non-AM, soft-charging topology is also proposed in [15], that increases the effective flying capacitance density, leading to a boost for both high-and low power-densities. Said topology is, however, based on a Series-Parallel toplogy which is inherently less efficient at low power-densities, and the relative improvement is limited to a factor of two.

This paper is organized as follows. In Section II, two soft-charging techniques that increase the effective flying

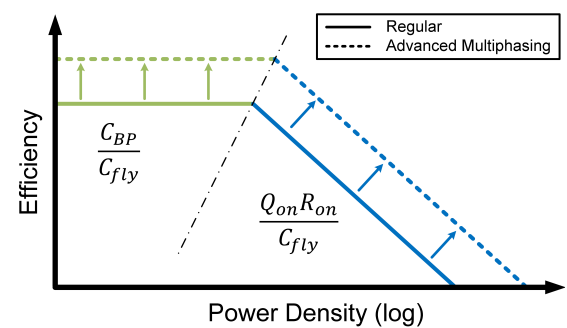

Fig. 2. Effect of Avanced Multiphasing on the monolithic SC converter design space.

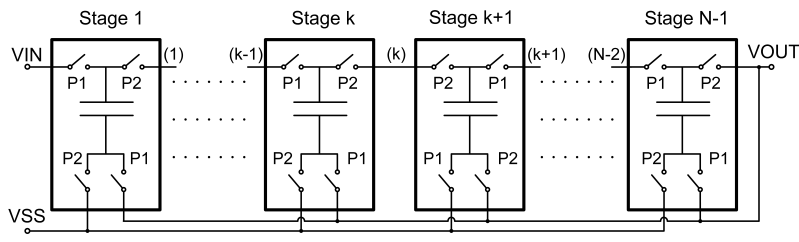

Fig. 3. A regular N:1 Dickson SC converter.

capacitance, called Stage Outphasing (SO) and Multiphase Soft-Charging (MSC), are introduced. Section III goes into more detail how these techniques can be efficiently implemented using an example implementation. Measurement results, showing the effectiveness of SO and MSC in a realized converter, are discussed in Section IV. Finally, Section V highlights the important conclusions of this work in a brief summary.

\section{Stage Outphasing And Multiphase SOFT-CHARGING}

While the proposed techniques and concepts can be applied to multiple SC topologies, for the brevity of this work, the discussion is limited to their application in a Dickson converter, shown in Fig. 3, due to its excellent properties for monolithic integration [16]. An additional advantage of the Dickson topology is that it can be extended to gearbox or multi-ratio use with relative ease thanks to the Folding concept [17]. Both techniques presented in this paper are fully compatible with said concept.

\section{A. Regular Converter}

In a regular N:1 Dickson converter, there are a total of $\mathrm{N}-1$ stages. If $V_{\text {out }}$ is close to the technology's $V_{D D}$, the top-side switches are usually implemented as two stacked transistors to avoid using less-efficient I/O devices. This also leads to the creation of intermediate nodes $(\mathrm{k})$, which are topologically speaking DC nodes and can be used as voltage rails.

All stages of a Dickson converter are identical, except for the fact that they are shifted in the voltage domain, and that adjacent stages run in anti-phase of one another. 


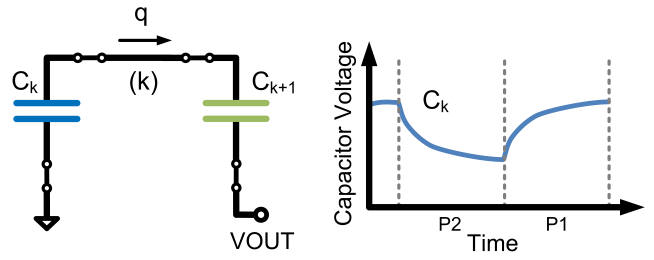

Fig. 4. Charge transfer between two adjacent stages of a regular Dickson converter. $C_{k}$ and $C_{k+1}$ are the flying capacitances of stages $\mathrm{k}$ and $\mathrm{k}+1$ respectively, $q$ is the transferred charge.

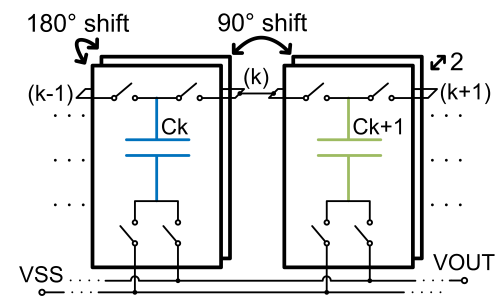

Fig. 5. Application of Stage Outphasing to a Dickson converter.

Thanks to this symmetry, the analysis of a Dickson converter can be simplified by looking at just a single stage's charging- and discharging state, and extrapolating these results to the rest of the converter. Alternatively, because of the same symmetry discussed above, it is possible to look at the charge transfer(s) between two arbitrary stages. After all, in a single charge transfer, one stage is charged and another is discharged, giving the same information as the analysis of just one stage.

Figure 4 portrays such a charge transfer. Here a certain amount of charge, $q$ is transferred from stage $\mathrm{k}$ to stage $\mathrm{k}+1$. This charge transfer generates a voltage swing across both capacitor's terminals, $\Delta V$, which is proportionate to $q$, and causes charge-sharing losses $P_{c s}$ :

$$
P_{c s} \propto \frac{q^{2}}{C_{k}} .
$$

\section{B. Stage Outphasing}

With SO, each stage is divided into two cells that connect to the same nodes, (k-1) and (k), but run in antiphase, as shown in Fig. 5. This way, for every stage, there is always one (and exactly one) cell that connects to each of the adjacent nodes. By phase-shifting adjacent stages $90^{\circ}$, a single cell connects to two cells of the adjacent stage over the duration of a charging- or discharging state, splitting said state up into two phases, as depicted in Fig. 6. The key here is that, in steady state, the charges that are transferred in each phase, $q_{1}$ and $q_{2}$, are equal in size. This can be explained as follows. Let $V_{A}$ and $V_{B}$ be the top-plate (TP) voltages of $C_{k}^{1}$ and $C_{k+1}^{1}$ respectively at the start of phase one, and $V_{C}$ be their TP voltage at the end of the same phase. Under
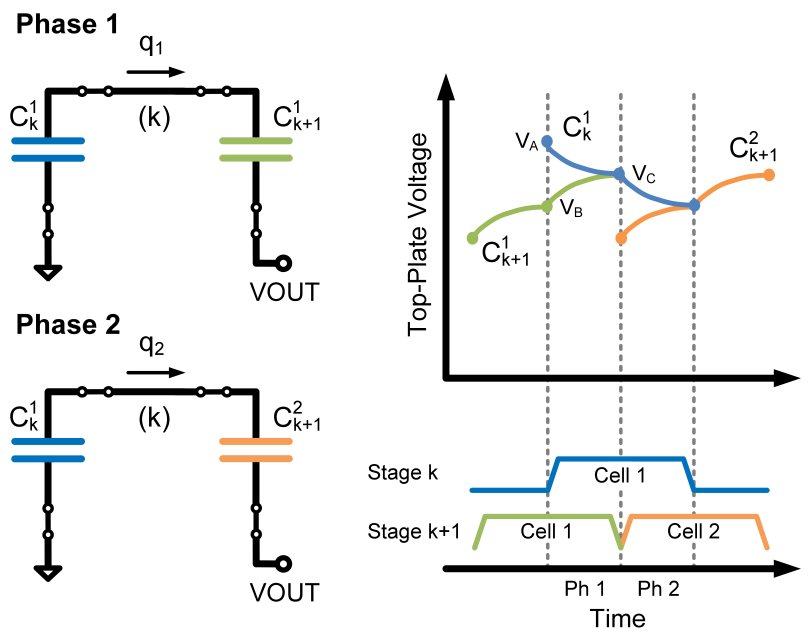

Fig. 6. Charge transfers between two adjacent stages of a Dickson converter using Stage Outphasing.

the assumption that $C_{k}^{1}$ and $C_{k+1}^{1}$ are equal in size, $V_{C}$ satisfies

$$
V_{A}-V_{C}=V_{C}-V_{B}
$$

Now, if the system is in steady-state and the cells of each stage are phase-shifted versions of each other, the TP voltage of $C_{k+1}^{2}$ at the end of phase 2 will also be $V_{B}$. Furthermore, because the TP of $C_{k+1}^{2}$ is connected to the TP of $C_{k}^{1}$ in this phase, $V_{B}$ also corresponds to the convergence voltage of the latter. From (2) then follows that $q_{1}=q_{2}$.

For the same total charge transferred $q=2 q_{1}$, the charge-sharing losses are described by

$$
\begin{aligned}
P_{c s} & \propto 2 C_{k}\left(\frac{\Delta V}{2}\right)^{2} \\
& \propto \frac{q^{2}}{2 C_{k}},
\end{aligned}
$$

which is a factor of 2 improvement compared to the regular Dickson case. Alternatively, twice the charge can be transferred at the same conduction efficiency, equivalent to doubling the flying capacitance.

Note that while SO does decrease the charge-sharing losses, the intermediate nodes, (k), are not topologically DC. This means that any parasitic capacitance (e.g. well capacitance) on these nodes will introduce losses due to their inherent voltage swing. In practice, however, the voltage swing and capacitance on these nodes is small enough that these losses can be neglected.

Also, SO does not add any additional power transistors to the topology. In this sense, at least from a topology perspective, the gain that SO provides comes with no notable downsides. This is due to the fact that there 


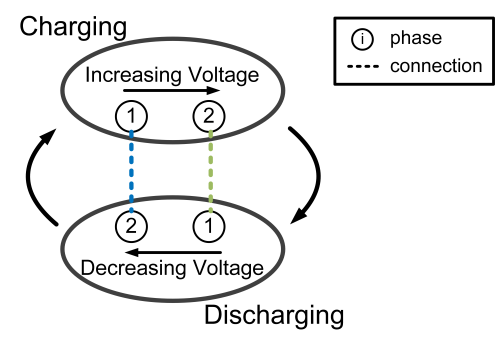

Fig. 7. State diagram of a stage outphasing Dickson converter.

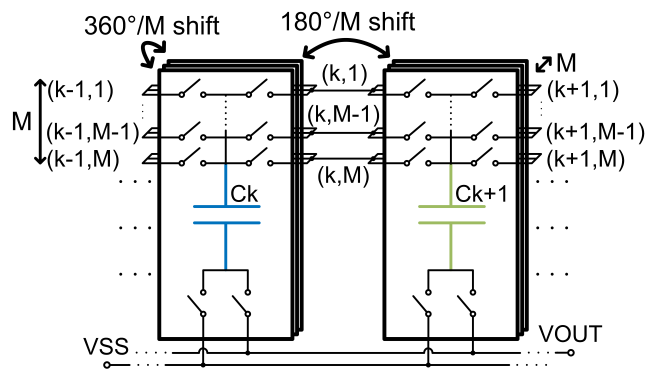

Fig. 8. Application of Multiphase Soft-Charging to a Dickson converter with a factor of $\mathrm{M}$.

is already some redundancy present in the form of the stacked top-side transistors in the regular topology.

What might not be obvious from the above discussion is why stage outphasing works. To answer this, consider the state diagram of a stage outphasing converter shown in Fig. 7. As can be seen, a cell in the first phase of the charging state, which has presumably the lowest voltage of all charging cells, connects to the cell in the last phase of the discharging state which in turn has the lowest voltage of all discharging cells. Similarly, the discharging cell of the first phase connects to the charging cell of the last phase, both cells having the highest voltage of their respective state. In other words, each charge transfer takes place between cells whose voltages match each other's most closely, minimizing the charge-sharing losses.

\section{Multiphase Soft-Charging}

From Fig. 7 it is easy to conceive a state diagram which has even lower charge-sharing losses. Simply by adding more phases to both the charging- and discharging state, the total charge transfer will take place in more steps.

To realize this, the number of phases of the converter itself needs to be increased. This is precisely what multiphase soft-charging does, shown in Fig. 8. Here, each intermediate node is split into $\mathrm{M}$ seperate nodes, $(\mathrm{k}, 1)$ to $(\mathrm{k}, \mathrm{M})$, and a switch is added from each cell to all adjacent nodes. In addition, each stage is split into $\mathrm{M}$ cells which are phase-shifted $360^{\circ} / \mathrm{M}$ and adjacent stages
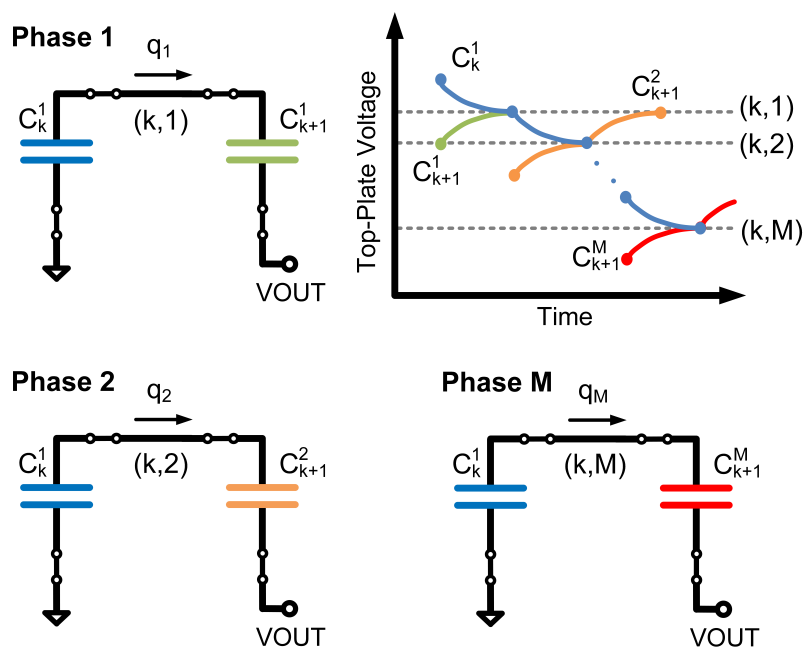

Fig. 9. Charge transfers between two adjacent stages of a Dickson converter using Multiphase Soft-Charging.

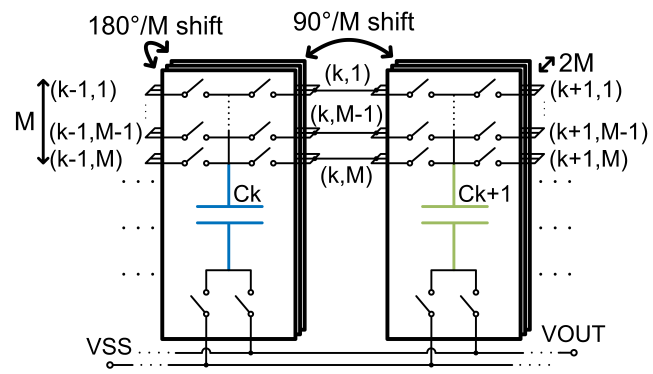

Fig. 10. Application of a combination of Stage Outphasing and Multiphase Soft-Charging to a Dickson converter with a factor of $\mathrm{M}$.

are further shifted $180^{\circ} / \mathrm{M}$. A full cycle takes a total of $2 \mathrm{M}$ phases which are split evenly in the charging and discharging state.

Figure 9 depicts the charge transfers of a discharging cell in a converter using MSC. Using the same reasoning as in Section II-B, it can be shown that in steady-state the charge transferred in two adjacent phases is equal in size. Extrapolating this result, the charge transfers of all phases in a state must also be equal. Moreover, this implies that the voltages of nodes $(\mathrm{k}, 1)$ to $(\mathrm{k}, \mathrm{M})$ are equidistant. Normalizing for the total charge transferred, the total charge-sharing losses of a flying capacitor over a full charge/discharge are consequently proportionate to

$$
P_{c s} \propto \frac{q^{2}}{M C_{k}} .
$$

Thus, MSC enables a scalable improvement of the charge-sharing losses by changing the factor $\mathrm{M}$.

Stage outphasing and multiphase soft-charging are not mutually exclusive and can be combined with little effort. As shown in Fig. 10, compared to a design using only MSC, the number of cells per stage simply needs 
to be doubled, and the phase shift between cells of the same stage and stages themselves halved. As one would expect, the following then holds

$$
P_{c s} \propto \frac{q^{2}}{2 M C_{k}}
$$

\section{Full Soft-Charging Converter}

By definition, a transfer between a single capacitor and a fixed DC voltage (e.g. $V_{\text {out }}-V_{\text {ss }}$ or $V_{\text {in }}-V_{\text {out }}$ ) can not be soft-charged. Both SO and MSC can thus only be applied to charge transfers between capacitors. For a Dickson converter, though, all but two transfers can be soft-charged, regardless of the number of stages. Because the number of stages determines the VCR, applying SO and MSC to the Dickson topology consequently gets better for increasing VCR.

Both SO and MSC are techniques that in essence modify a two-phase SC topology to a more efficient version using multiple phases. The performance of a SC topology is determined by its topological factors, $K_{c}$ and $K_{s}$, which are proportionate to the topology's conduction losses in the slow-switching limit (SSL) and fast-switching limit (FSL) respectively [10], [18]. The smaller these factors, the more efficient the topology utilizes its capacitors or switches. Given a topology, the values of $K_{c}$ and $K_{s}$ depend on the relative sizing of the capacitors and switches respectively. By sizing these components according to the amount of charge transferred, the topological factors can be optimized [18].

To derive $K_{c}$ for a Dickson converter using SO and/or MSC, there are multiple options. A first one is to use the general multiphase SC model described in [19], which has the downside of requiring a lengthy calculation. A much more simple and elegant way is to start with the result for a regular N:1 Dickson converter with equally, and thus optimally [18], sized capacitors:

$$
K_{c, \text { regular }}=\left(\frac{N-1}{N}\right)^{2}
$$

Assume a Dickson converter with $\mathrm{S}$ soft-charging steps for all capacitor-capacitor charge transfers, which means either MSC is used with $\mathrm{M}=\mathrm{S}$, or a combination of $\mathrm{SO}$ and MSC is used with $2 \mathrm{M}=\mathrm{S}$. In this case, two charge transfers, the charging of the first stage by $V_{\text {in }}-V_{\text {out }}$ and the discharging of the final stage to $V_{\text {out }}-V_{s s}$ remain unchanged, while the $2(\mathrm{~N}-2)$ other transfers' losses are reduced by a factor of S. Keeping in mind that there are a total of $2(\mathrm{~N}-1)$ charge transfers for a regular N:1 Dickson converter, each of which contributes equally to $K_{c, \text { regular }}$, the soft-charged topological factor can be written as

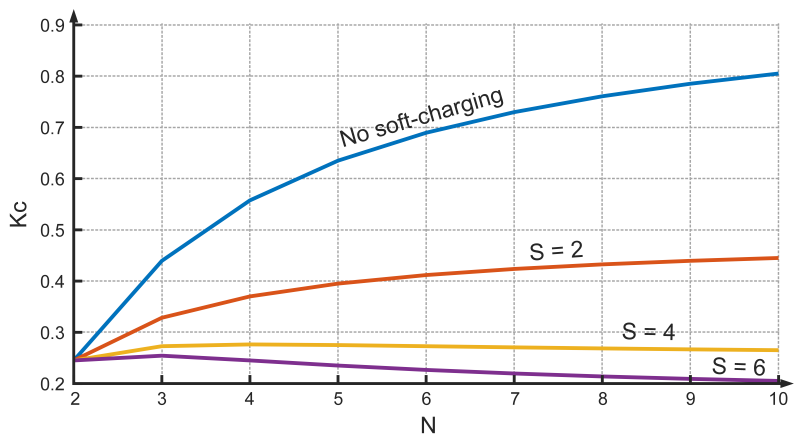

Fig. 11. Scaling of a N:1 Dickson converter's $K_{c}$ versus $\mathrm{N}$ without soft-charging, and with soft-charging using a selection of soft-charging factors, $\mathrm{S}$.

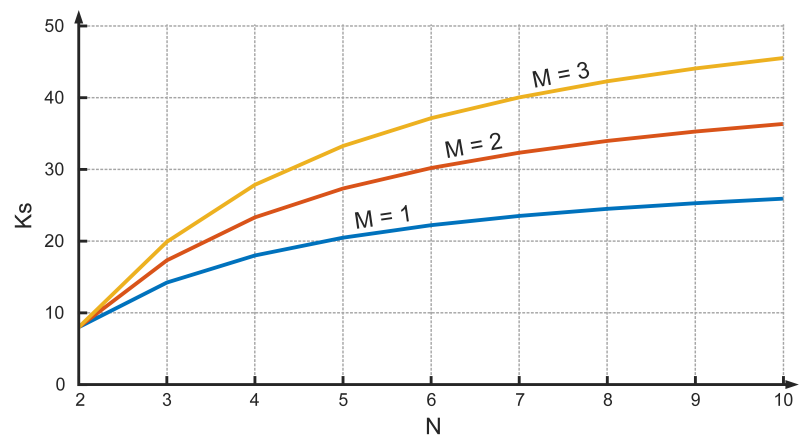

Fig. 12. Scaling of a N:1 Dickson converter's $K_{s}$ versus N without MSC, and with MSC using a selection of factors, M.

$$
\begin{aligned}
K_{c, s c} & =\frac{K_{c, \text { regular }}}{2(N-1)}\left(2+\frac{2(N-2)}{S}\right) \\
K_{c, s c} & =\frac{N-1}{N^{2}}\left(1+\frac{(N-2)}{S}\right) .
\end{aligned}
$$

Figure 11 shows how $K_{c}$ scales with respect to $\mathrm{N}$ and the soft-charging factor, $\mathrm{S}$, and includes a comparison to the regular Dickson converter. For increasingly large N, $K_{c}$ tends towards $S^{-1}$. Furthermore, the largest relative gains are to be had for smaller S. As expected though, these gains do get more significant for larger VCR's.

While SO does not add any power transistors, MSC does require them, which will lead to an increase of the topology's $K_{s}$. Using the model of [19], a general solution for $K_{s}$ was found using optimal relative sizing of the switches:

$K_{s, m s c}=\frac{4}{N}(2 \sqrt{M}(N-2)+2 N)\left(1+\frac{(N-2)}{N} \sqrt{M}\right)$

The scaling of $K_{s}$ is portrayed in Fig. 12. As can be seen, increasing $M$ does have a notable impact on the FSL losses. Moreover, the absolute increase is relatively 


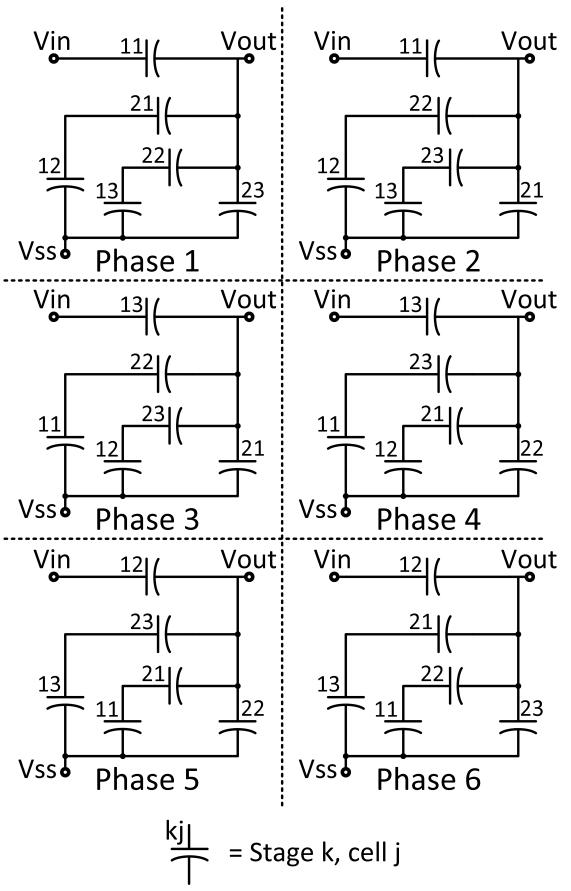

Fig. 13. Phase diagram of the implemented 3:1 Dickson converter using MSC and SO.

constant regardless of $\mathrm{M}$. Comparing these results with Fig. 11, it is clear that after a certain point, increasing $\mathrm{M}$ will add more losses in the switches than are reduced in the capacitors. What exactly this optimum is, however, depends heavily on the converter's VCR and power density, and a number of technology parameters.

\section{IMPLEMENTATION}

\section{A. System}

The proposed techniques of stage outphasing and multiphase soft-charging are implemented in a 3:1 Dickson converter with $\mathrm{M}=2$. Using (9) one can calculate that this leads to a $60 \%$ higher effective capacitance density. Because a 3:1 converter only has two stages, each stage has a soft-charged- and a hard-charged state. The hardcharged state, however, does not require the same amount of time to complete as the soft-charged state, which is spread over multiple phases. Therefore, the number of phases of the hard-charged state is reduced from four to two in this implementation. This has the advantage of also reducing the total number of phases and cells per stage to six and three respectively, which in turn reduces frequency and margin overhead. The resulting phase diagram of the implemented converter is shown in Fig. 13.

Figure 14 gives an overview of the full converter, including the controller. Because of SO, only half of the phases are used in each stage. A second core can

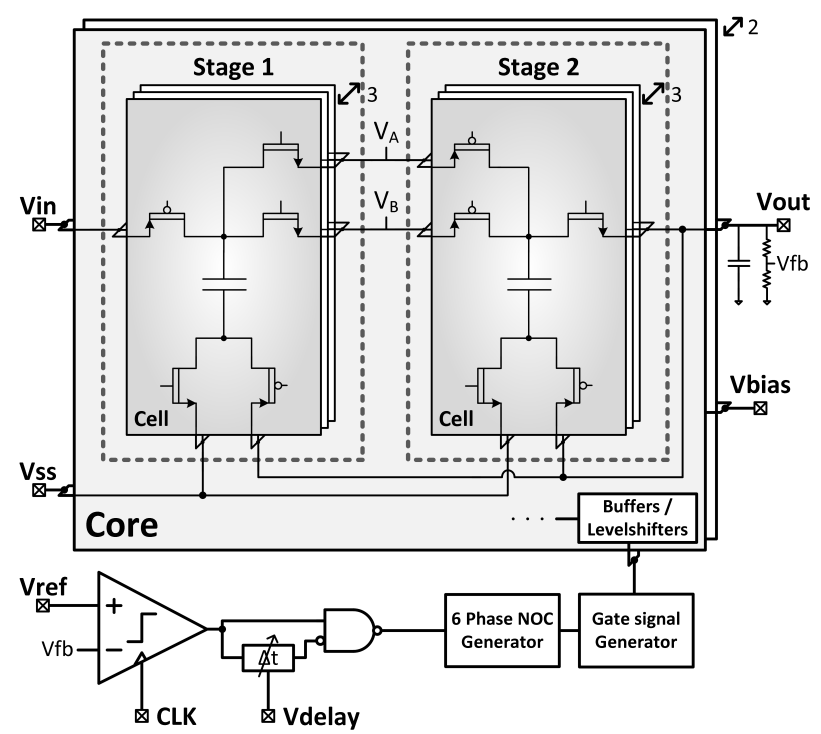

Fig. 14. System overview of the 3:1 converter, showing the controller and transistor-level implementation of the converter cores.

consequently be added that runs in anti-phase relative to the first, and uses the other half of the phases. As a result, the amount of charge that is transferred to the output each switch event is equalized, and the output ripple is subsequently reduced. In addition, a small amount of decoupling capacitance, equal to approximately $9 \%$ of the total flying capacitance is integrated for the output terminal.

The hysteretic controller runs at a clock frequency of $1.6 \mathrm{GHz}$, which means that the converter itself runs at $267 \mathrm{MHz}$. A rising edge-detect circuit with a tunable delay cell generates a pulse with a variable width which is in turn used to clock a 6 phase ring-connected one-hotcoded oscillator based on SR flipflops. The width of the pulse ultimately determines the width of the dead time between every phase. The six non-overlapping clock (NOC) phases are then used to generate the required gate signals. Due to the relatively small amount of unique gate signals, this is done centrally. In fact, only the capacitive levelshifters [12] and buffers are placed close to each cell. Even though the intermediate nodes, $V_{A}$ and $V_{B}$, are not DC because of SO, they are used to power the top-side buffers and power transistors with little effect on the soft-charging operation of the converter.

\section{B. Capacitor Implementation}

The flying capacitors are implemented using a combination of MOM- and MOS-capacitors, with thick-oxide devices used in the first stage due to their higher voltage rating. MOS-capacitors, while having a high capacitance density, also have a notoriously high parasitic coupling due to their proximity to the substrate. To limit the 


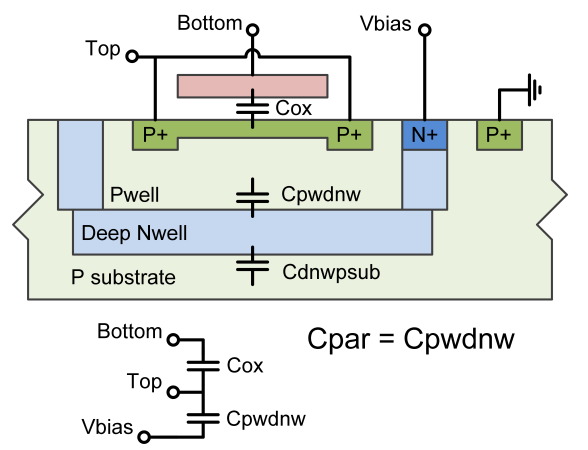

(a)

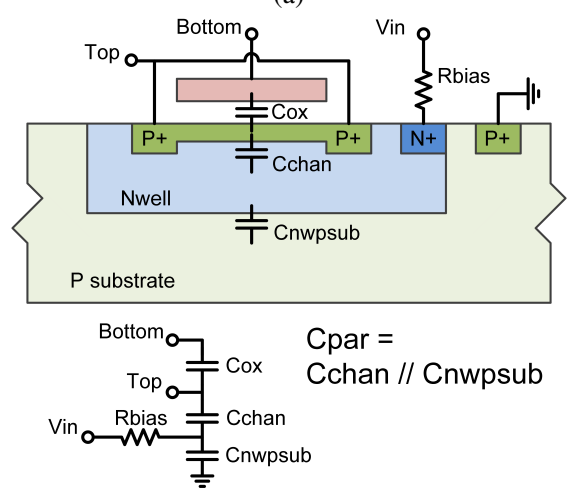

(b)

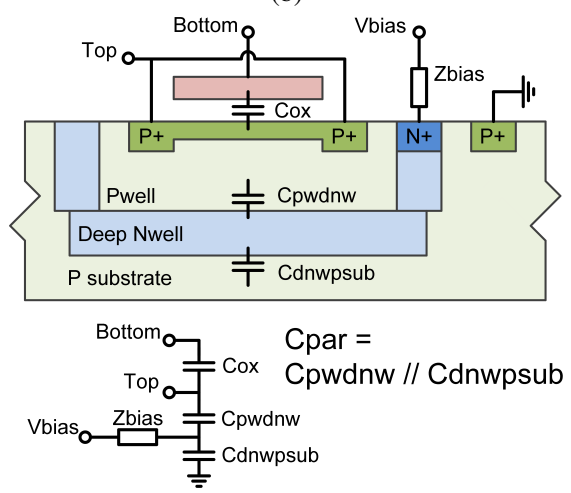

(c)

Fig. 15. Schematic representation of the biasing techniques discussed in (a) [20], (b) [21] and (c) the one used in this work.

influence of the latter, it is possible to bias the wells of the MOS-capacitors with a suitable bias circuit [22]. Multiple converters in literature have implemented such techniques [12], [20], [21], [23]. Figure 15 shows a schematic comparison of a selection of these to the implementation used in this work.

The accumulation-based capacitor of [20] (Fig. 15a) has the advantage of having a smaller parasitic junction capacitor, $C_{p w d n w}$, over the P-well to deep N-well (DNW) junction, compared to the channel capacitor, $C_{\text {chan }}$, of the inversion-based capacitor used in [21] (Fig. $15 b)$. This is due to the fact that the latter has higher doping concentrations. The implementation of [21], on the other hand, does bias the N-well using a sufficiently

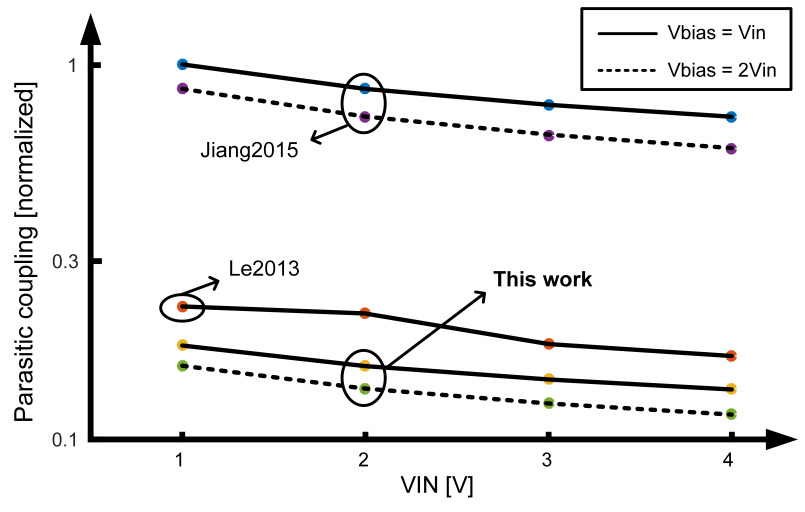

Fig. 16. Comparison of the simulated parasitic coupling of the used implementation to literature [20], [21].

large resistor, causing the channel capacitor to effectively be placed in series with the N-well to substrate junction capacitor, $C_{n w p s u b}$ and leading to significant reduction in parasitics.

The proposed implementation combines the advantages of both to minimize the effective parasitic coupling as much as possible. The result is an accumulationbased capacitor that uses a dedicated high voltage bias, $V_{\text {bias }}$, to bias the DNW. Furthermore, by using two front-to-front diode-connected PMOS devices, the highimpedance is realized using at least 100 times less area overhead than the approach in [21]. Figure 16 compares the parasitic coupling of the used implementation to the state-of-the-art. As can be seen, the used MOS capacitors have significantly lower coupling. For a bias voltage equal to the converter's input voltage, an improvement of $18 \%$ is witnessed, while higher relative bias voltages can push this improvement to $30 \%$ and more. Overall, thanks to the proposed implementation, the ratio of parasitic- to flying capacitance is made smaller than $1 \%$.

Note also that all of the shown capacitor implementations have their parasitic coupling on the flying capacitor's top plate. This has the inherent advantage that the parasitic capacitor acts as an extra parallel 1:1 converter which supplies power to the load, rather than an additional load which needs to be supplied by the SC converter itself if the parasitic coupling were present on the bottom-plate [24].

\section{Measurements And Realization}

The design is realized in a $28 \mathrm{~nm}$ baseline CMOS process using a total flying capacitance of $1.5 \mathrm{nF}$. Figure 17 shows the microchip which measures $0.260 \times 0.448$ $\mu \mathrm{m}^{2}$. As discusses in the previous section, the capacitors of the first stage are partly implemented using thickoxide MOS capacitors and take up a larger area as a result. 


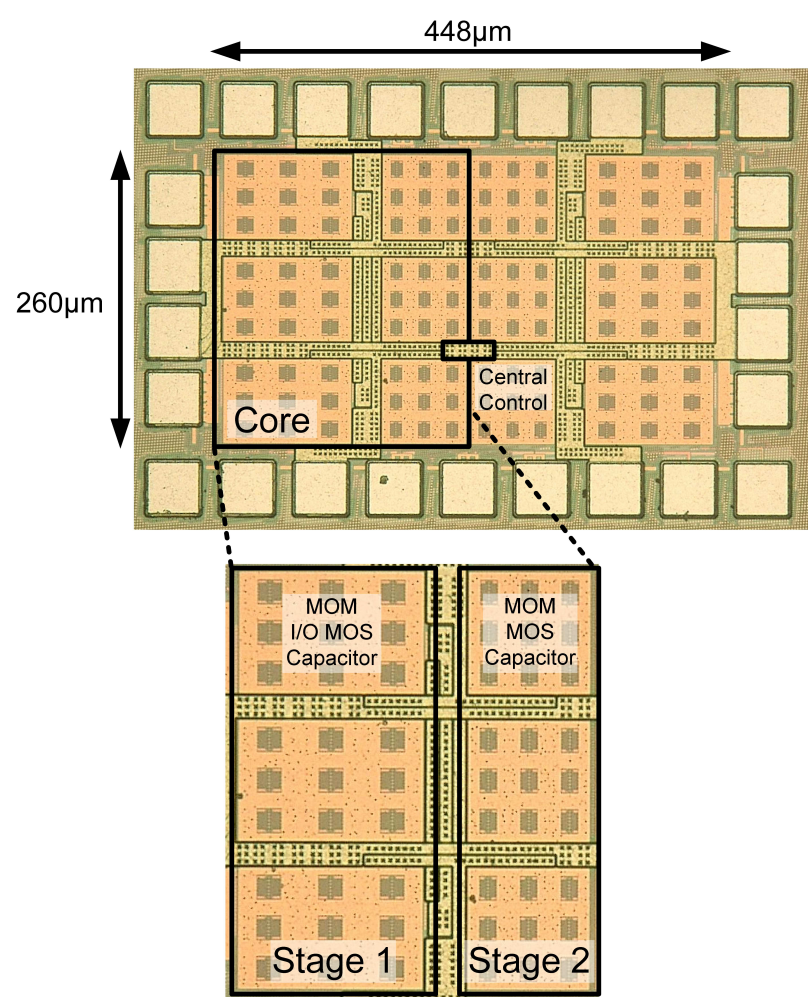

Fig. 17. Annotated micrograph of the monolithic 3:1 SC Dickson converter using SO and MSC, measuring $0.117 \mathrm{~mm}^{2}$ without bond pads.

Because of the fact that this implementation uses a total of six phases of which four are used to soft-charge either the charging or discharging state, every cell of stage one will connect to a certain cell of the second stage two times instead of just once over a full cycle. To minimize the resistive losses in the interconnects between cells and stages, these pairs of cells are positioned closest to each other.

The generation of $V_{\text {bias }}$ is not realized on-chip in this design. For all below measurements a voltage of $8.5 \mathrm{~V}$ was supplied externally, drawing less than 60nA at an ambient temperature of approximately $295 \mathrm{~K}$. While this voltage is much higher than the technology's nominal voltage, it is still well below the junction's breakdown voltage [25], [26].

\section{A. Intermediate Nodes}

To verify the working principle of MSC, the converter is measured under open-loop operation with increasing load currents. The results hereof are demonstrated in Fig. 18. The theoretical prediction is done using a chargeanalysis of the topology and states that, given $V_{i n}$ and $V_{\text {out }}, V_{A}$ and $V_{B}$ can be determined as

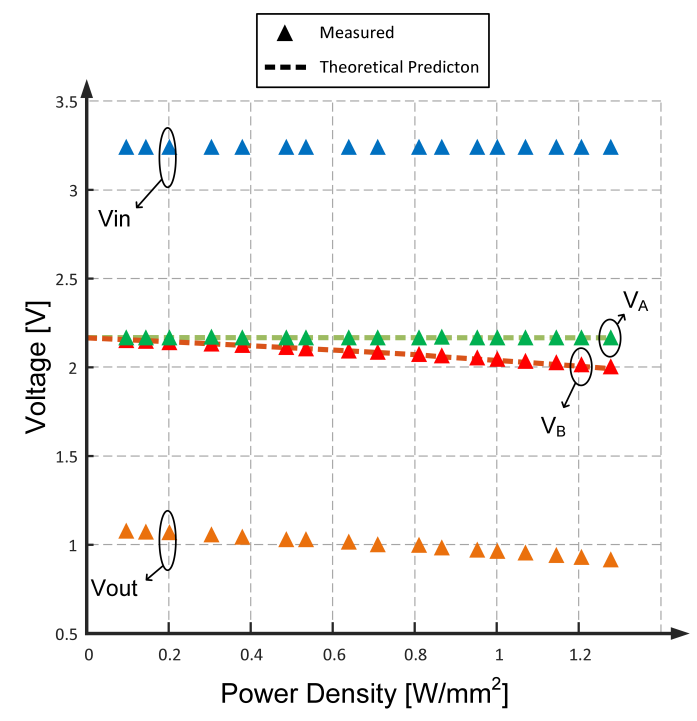

Fig. 18. Comparison of measured DC voltages of intermediate nodes, $V_{A}$ and $V_{B}$, under open-loop operation with $f_{c l k}=1.3 \mathrm{GHz}$ and $V_{i n}=3.25 \mathrm{~V}$, to theoretical predicted values based on $V_{\text {in }}$ and $V_{\text {out }}$ measurements

$$
\begin{aligned}
V_{A} & =\frac{2}{3} V_{i n} \\
V_{B} & =\frac{1}{3} V_{\text {in }}+V_{\text {out }},
\end{aligned}
$$

which can be rewritten to

$$
V_{A}-\frac{V_{\text {in }}+V_{\text {out }}}{2}=\frac{V_{\text {in }}+V_{\text {out }}}{2}-V_{B}
$$

As can be seen from Fig. 18, the intermediate nodes are pulled apart in order to supply more charge each clock cycle. At the same time, in agreement to (13), they remain symmetrical with respect to the average of $V_{\text {in }}$ and $V_{\text {out }}$, which implies that the charge transfers of both MSC phases are approximately equal. Overall, the measured behavior of the intermediate nodes matches the theoretical prediction of (11) and (12) with less than $1 \%$ error.

\section{B. Efficiency}

The converter's closed-loop efficiency is shown in Fig. 19, which is measured using Kelvin contacts and includes all system losses. A peak efficiency of $82 \%$ is achieved at power densities of $0.65-1.1 \mathrm{~W} / \mathrm{mm}^{2}$ or output powers of $76-126 \mathrm{~mW}$. Thanks to the hysteretic controller, the input-referred quiescent current of the converter is approximately $0.9 \mathrm{~mA}$, leading to a wide efficient output power range. 


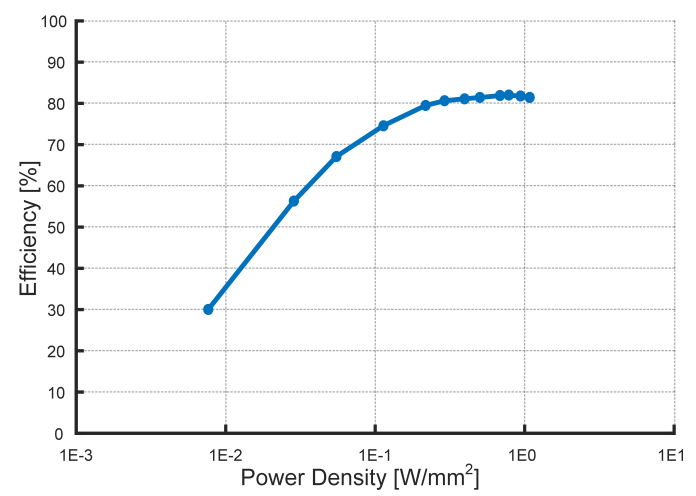

Fig. 19. Measured full-system closed-loop efficiency versus output power-density for $f_{c l k}=1.6 \mathrm{GHz}, V_{\text {in }}=3.2 \mathrm{~V}$ and $V_{\text {out }}=0.95 \mathrm{~V}$.

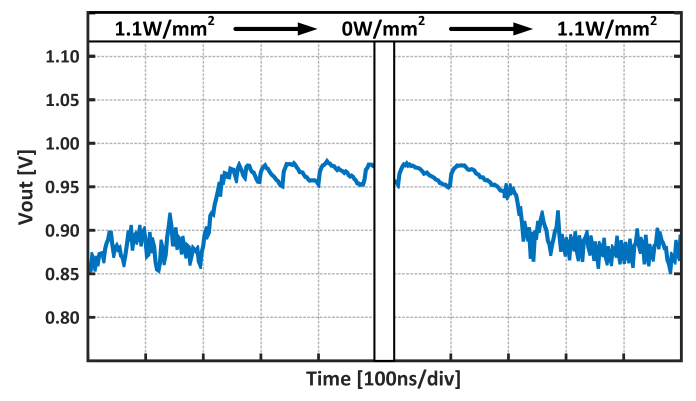

Fig. 20. Measured worst-case load-step transient response with $f_{c l k}=1.6 \mathrm{GHz}, V_{\text {in }}=3.2 \mathrm{~V}$ and $V_{\text {ref }}=0.95 \mathrm{~V}$.

\section{Controller}

Figure 20 demonstrates the operation of the hysteretic controller as it is tested under worst-case load-regulation conditions. For this measurement, the load power is switched from the maximum load power of $126 \mathrm{~mW}$ to zero and back with a transient time of $18 \mathrm{~ns}$. As can be seen, no notable droop or overshoot is present.

\section{Comparison}

The presented work is compared to the state-of-the-art of fully integrated SC DC-DC converters in Fig. 21 and Table I. Thanks to the presented soft-charging techniques and the capacitor implementation, the realized converter achieves a significantly higher efficiency-power-density combination than any other published planar bulk SC converter, and also outperforms designs using SOI or TriGate technologies. Relative to the current state-of-theart of planar bulk CMOS SC converters, the presented work has either 29x higher power-density for the same efficiency, or 3x lower losses for the same power-density.

While efficiency versus power-density comparisons such as the one portrayed in Fig. 21 are useful, they do not show the full picture. In particular, no information regarding the voltage conversion ratio is included, even though this is a crucial factor for many applications,

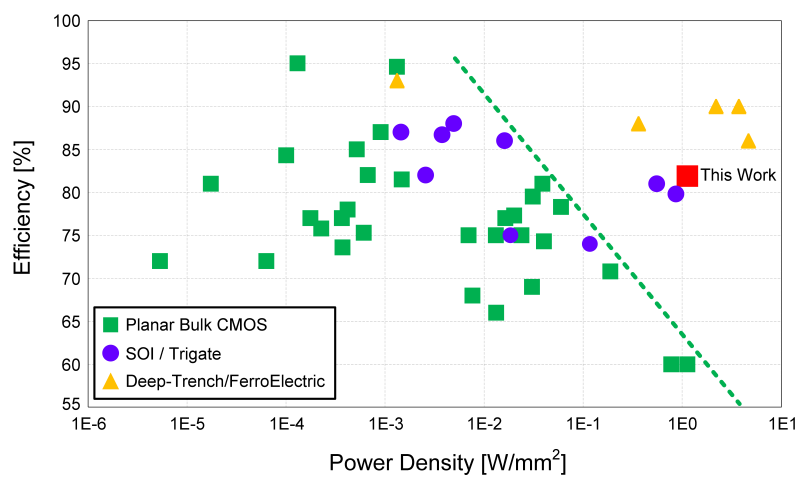

Fig. 21. Extensive efficiency versus power-density comparison of the presented converter to the state-of-the-art of fully integrated SC converters, based on [8]. The dotted line represents the current stateof-the-art for planar bulk CMOS designs.

including the reduction of PDN losses discussed in Section I. Therefore, two Figures-of-Merit (FoMs) are proposed that incorporate the VCR.

The PDN FoM gives an idea how useful a given converter is to reduce PDN losses for an integrated system and is defined as the lowest PDN losses for which the addition of the converter still improves the system efficiency:

$$
F_{O M_{P D N}}=\frac{1-\eta}{\eta-V C R}
$$

with $V C R$ the actual output-to-input voltage ratio, not the topology VCR, $V C R_{\text {top }}$. The High-Density (HD) FoM is more general and represents the converter's efficiency, VCR and power density in one figure, by comparing the converter to theoretical performance limits of a two-phase SC converter at high power densities, $P_{\text {dens }}$ :

$$
F_{o M_{H D}}=\frac{P_{D C D C}}{\sqrt[3]{\left(1-V C R_{t o p}\right)^{4} P_{d e n s}}},
$$

Here, $P_{D C D C}$, are the converter losses divided by its output power. The derivation of both FoMs is added in the Appendix to this paper. Figure 22 compares the presented work to the state-of-the-art using the PDNand HD FoM. As can be seen, the presented work performance significantly better compared to both planar bulk and SOI/Trigate designs, thanks to its high efficiency, power-density and voltage conversion ratio. In fact, the presented work achieves results that are nearly comparable to designs using expensive DeepTrench capacitors, which have more than 20 times the capacitance density and are far from common in today's technology nodes. 
TABLE I

COMPARISON TO STATE-OF-THE-ART.

\begin{tabular}{lcccc}
\hline Item & This work [27] & {$[13]$} & {$[12]$} & {$[9]$} \\
\hline Technology & $\mathbf{2 8 n m}$ & $32 \mathrm{~nm}$ & $90 \mathrm{~nm}$ & $32 \mathrm{~nm}$ SOI \\
Capacitors & MOM+MOS & MOM & MOS & MOM+MOS \\
$V C R_{\text {top }}$ 's & $\mathbf{3 : 1}$ & $1: 2$ & $2: 1$ & $3: 22: 13: 1$ \\
$V_{\text {in }}[V]$ & $\mathbf{3 . 2}$ & 1 & $2.4-2.6$ & 2 \\
$V_{\text {out }}[V]$ & $\mathbf{0 . 9 5}$ & 1.5 & $0.9-1.3 *$ & $0.5-1.15$ \\
$P_{\text {dens,quoted }}\left[\mathrm{mW} / \mathrm{mm}^{2}\right]$ & $\mathbf{1 . 1}$ & 1.1 & 0.77 & 0.86 \\
$\eta$ @ $P_{\text {density,quoted }}$ & $\mathbf{8 2 \%}$ & $60 \%$ & $60 \%$ & $79.8 \%$ \\
Closed Loop? & Yes & Yes & No & No \\
Area $\left[m m^{2}\right]$ & $\mathbf{0 . 1 1 7}$ & 0.067 & 2.14 & 0.378 \\
$V C R_{\text {top }} @ P_{\text {dens, quoted }}$ & $\mathbf{3 : 1}$ & $1: 2$ & $2: 1$ & $2: 1$ \\
$P_{D C D C} @ P_{\text {dens }, q u o t e d}$ & $\mathbf{2 2 \%}$ & $67 \%$ & $67 \%$ & $25 \%$ \\
PDN FoM** & $\mathbf{3 4 . 5 \%}$ & N/A & $183.2 \%$ & $56.0 \%$ \\
High Density FoM** $\left[\left(m W / m^{2}\right)^{-\frac{1}{3}}\right]$ & $\mathbf{0 . 3 7}$ & 1.64 & 1.83 & 0.67 \\
\hline *estimate based on graphs & & & & \\
** See Appendix, smaller is better & & & &
\end{tabular}

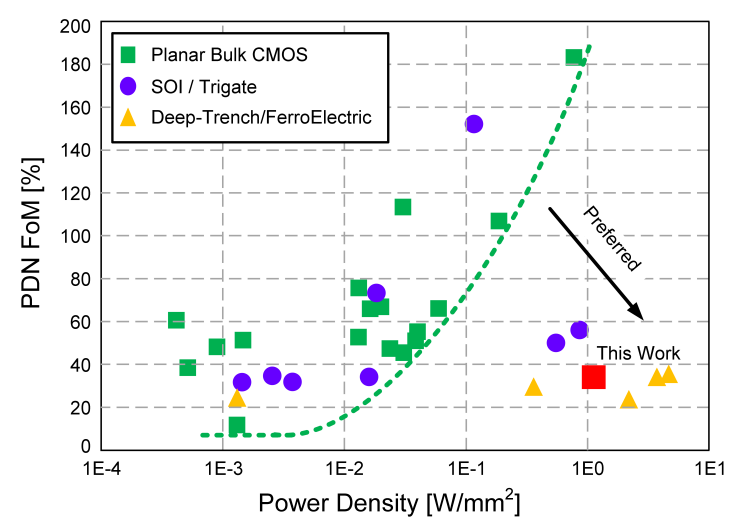

(a)

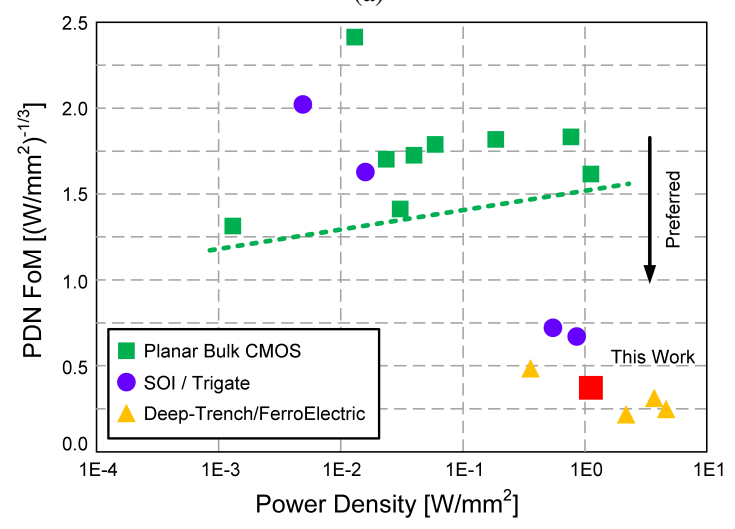

(b)

Fig. 22. Comparison of the presented work to the state-of-the-art using (a) the power delivery network- (PDN) and (b) the High-Density Figure-of-Merit. The dotted line represents the current state-of-the-art for planar bulk CMOS designs.

\section{CONCLUSion}

In this work, the need for high efficiency, large VCR and high power-density fully-integrated DC-DC converters using common technologies was discussed, together with the difficulty of achieving this due to the limited capacitance density available in modern technology processes. Two techniques, called Stage Outphasing and Multiphase Soft-Charging, were introduced that make use of the advanced multiphasing concept to soft-charge charge transfers between flying capacitors. As such the effective flying capacitance can be increased. The techniques were analyzed for a Dickson SC converter and their impact on the topological parameters were discussed. A 3:1 Dickson SC converter was realized that implements these techniques to achieve a stateof-the-art $1.1 \mathrm{~W} / \mathrm{mm}^{2}$ power-density and $82 \%$ efficiency combination using common capacitor technologies.

\section{APPENDIX}

Derivation of power delivery network (PDN) FoM: Consider the two systems of Fig. 1. Without an integrated converter, there are a certain amount of PDNinduced losses, $P_{P D N}^{(1)}$ which are proportionate to the current drawn by the load, $I_{\text {Load }}$, and normalized by the total load power. If an integrated converter is introduced, the PDN losses will be reduced by the converter's current conversion ratio:

$$
P_{P D N}^{(2)}=P_{P D N}^{(1)} \frac{I_{\text {in }}}{I_{\text {Load }}},
$$

where $I_{i n}$ is the input current of the converter. At the same time, the converter will add its own normalized 
losses, $P_{D C D C}$. The PDN FoM is defined as the breakeven point between both:

$$
\begin{aligned}
& P_{P D N}^{(1)}=P_{D C D C}+P_{P D N}^{(1)} \frac{I_{\text {in }}}{I_{\text {Load }}} \\
& P_{P D N}^{(1)}=F o M_{P D N}=\frac{1-\eta}{\eta-V C R} .
\end{aligned}
$$

The PDN FoM can also be written using the Efficiency Enhancement Factor (EEF) [1], which compares the input power of a converter to that of an LDO with the same VCR:

$$
F O M_{P D N}=\frac{P_{D C D C}}{E E F} .
$$

Derivation of High-Density (HD) FoM: For converters with a high output power-density [9], it can be shown that its normalized losses are proportionate to the topology's topological factors, $K_{c}$ and $K_{s}$, and the converter's power density, $P_{d e n s}$, as follows [9]:

$$
P_{D C D C} \propto \sqrt[3]{K_{c} K_{s} P_{\text {dens }}}
$$

Now, for a N:1 converter, the optimal value of $K_{c}$ and $K_{s}$ using limited voltage devices, are [18]:

$$
\begin{aligned}
& K_{s, o p t} \propto\left(\frac{N-1}{N}\right)^{2}=\left(1-V C R_{t o p}\right)^{2} \\
& K_{c, \text { opt }} \propto\left(\frac{N-1}{N}\right)^{2}=\left(1-V C R_{t o p}\right)^{2},
\end{aligned}
$$

with $V C R_{t o p}$ the topology's voltage conversion ratio. Combining above equations leads to

$$
P_{D C D C} \propto \sqrt[3]{\left(1-V C R_{t o p}\right)^{4} P_{\text {dens }}} .
$$

The FoM is then defined as the ratio of the converter's normalized losses to the right-hand side.

$$
F o M_{H D}=\frac{P_{D C D C}}{\sqrt[3]{\left(1-V C R_{t o p}\right)^{4} P_{d e n s}}} .
$$

\section{REFERENCES}

[1] M. Steyaert, T. Van Breussegem, H. Meyvaert, P. Callemeyn, and M. Wens, "DC-DC converters: From discrete towards fully integrated CMOS," in ESSCIRC (ESSCIRC), 2011 Proceedings of the, Sept 2011, pp. 42-49.

[2] M. Steyaert, F. Tavernier, H. Meyvaert, A. Sarafianos, and N. Butzen, "When hardware is free, power is expensive! Is integrated power management the solution?" in European SolidState Circuits Conference (ESSCIRC), ESSCIRC 2015 - 41st, Sept 2015, pp. 26-34.

[3] L. Chang, D. J. Frank, R. K. Montoye, S. J. Koester, B. L. Ji, P. W. Coteus, R. H. Dennard, and W. Haensch, "Practical Strategies for Power-Efficient Computing Technologies," Proceedings of the IEEE, vol. 98, no. 2, pp. 215-236, Feb 2010.
[4] "ITRS Reports," http://www.itrs2.net/itrs-reports.html, accessed: 2017-04-18.

[5] F. Carobolante, "Power Supply on Chip: from R\&D to commercial products," in International Workshop on Power Supply On Chip, 2014.

[6] T. M. Andersen, F. Krismer, J. W. Kolar, T. Toifl, C. Menolfi, L. Kull, T. Morf, M. Kossel, M. Brändli, P. Buchmann, and P. A. Francese, "A $4.6 \mathrm{~W} / \mathrm{mm} 2$ power density $86 \%$ efficiency on-chip switched capacitor DC-DC converter in $32 \mathrm{~nm}$ SOI CMOS," in Applied Power Electronics Conference and Exposition (APEC), 2013 Twenty-Eighth Annual IEEE, March 2013, pp. 692-699.

[7] Y. Lu, J. Jiang, and W. H. Ki, "A multiphase switched-capacitor DC-DC converter ring with fast transient response and small ripple," IEEE Journal of Solid-State Circuits, vol. 52, no. 2, pp. 579-591, 2017.

[8] M. Steyaert, N. Butzen, H. Meyvaert, A. Sarafianos, P. Callemeyn, T. Van Breussegem, and M. Wens, "DCDC performance survey," [Online]. Available: http://homes.esat.kuleuven.be/ steyaert/DCDC_Survey/DCDC_PS.html.

[9] H. P. Le, S. R. Sanders, and E. Alon, "Design Techniques for Fully Integrated Switched-Capacitor DC-DC Converters," IEEE Journal of Solid-State Circuits, vol. 46, no. 9, pp. 2120-2131, Sept 2011.

[10] N. Butzen and M. S. J. Steyaert, "Scalable Parasitic Charge Redistribution: Design of High-Efficiency Fully Integrated Switched-Capacitor DC-DC Converters," IEEE Journal of SolidState Circuits, vol. 51, no. 12, pp. 2843-2853, Dec 2016.

[11] N. Butzen and M. Steyaert, "A 94.6\%-Efficiency Fully Integrated Switched-Capacitor DC-DC Converter in Baseline 40nm CMOS using Scalable Parasitic Charge Redistribution," in 2016 IEEE International Solid-State Circuits Conference (ISSCC), Jan 2016, pp. 220-221.

[12] H. Meyvaert, G. V. Pique, R. Karadi, H. J. Bergveld, and M. S. J. Steyaert, "A Light-Load-Efficient 11/1 Switched-Capacitor DCDC Converter With $94.7 \%$ Efficiency While Delivering $100 \mathrm{~mW}$ at 3.3 V,' IEEE Journal of Solid-State Circuits, vol. 50, no. 12, pp. 2849-2860, Dec 2015.

[13] D. Somasekhar, B. Srinivasan, G. Pandya, F. Hamzaoglu, M. Khellah, T. Karnik, and K. Zhang, "Multi-phase 1ghz voltage doubler charge-pump in 32nm logic process," in 2009 Symposium on VLSI Circuits, June 2009, pp. 196-197.

[14] T. M. Andersen, F. Krismer, J. W. Kolar, T. Toifl, C. Menolfi, L. Kull, T. Morf, M. Kossel, M. Brändli, P. Buchmann, and P. A. Francese, "A sub-ns response on-chip switched-capacitor DCDC voltage regulator delivering $3.7 \mathrm{w} / \mathrm{mm} 2$ at $90 \%$ efficiency using deep-trench capacitors in 32nm SOI CMOS," in 2014 IEEE International Solid-State Circuits Conference Digest of Technical Papers (ISSCC), Feb 2014, pp. 90-91.

[15] S. R. Challa, D. Kastha, and A. Patra, "A Cascade Point of Load DC-DC Converter With a Novel Phase Shifted Switched Capacitor Converter Output Stage," IEEE Transactions on Power Electronics, vol. 31, no. 1, pp. 353-368, Jan 2016.

[16] H. Meyvaert, A. Sarafianos, N. Butzen, and M. Steyaert, "Monolithic switched-capacitor DC-DC towards high voltage conversion ratios," in 2014 IEEE 15th Workshop on Control and Modeling for Power Electronics (COMPEL), June 2014, pp. 1-5.

[17] A. Sarafianos and M. Steyaert, "Fully integrated wide input voltage range capacitive DC-DC converters: The folding dickson converter," IEEE Journal of Solid-State Circuits, vol. 50, no. 7, pp. 1560-1570, July 2015.

[18] M. D. Seeman, "A Design Methodology for Switched-Capacitor DC-DC Converters," Ph.D. dissertation, EECS Department, University of California, Berkeley, May 2009. [Online]. Available: http://www.eecs.berkeley.edu/Pubs/TechRpts/2009/EECS2009-78.html

[19] J. Delos, T. Lopez, E. Alarcón, and M. A. M. Hendrix, "On the modeling of switched capacitor converters with multiple outputs," in 2014 IEEE Applied Power Electronics Conference and Exposition - APEC 2014, March 2014, pp. 2796-2803.

[20] J. Jiang, Y. Lu, C. Huang, W. H. Ki, and P. K. T. Mok, "A 2-/3-phase fully integrated switched-capacitor DC-DC converter 
in bulk CMOS for energy-efficient digital circuits with $14 \%$ efficiency improvement," in 2015 IEEE International Solid-State Circuits Conference - (ISSCC) Digest of Technical Papers, Feb 2015, pp. 1-3.

[21] H. P. Le, J. Crossley, S. R. Sanders, and E. Alon, "A subns response fully integrated battery-connected switched-capacitor voltage regulator delivering $0.19 \mathrm{~W} / \mathrm{mm} 2$ at $73 \%$ efficiency," in 2013 IEEE International Solid-State Circuits Conference Digest of Technical Papers, Feb 2013, pp. 372-373.

[22] G. Villar-Pique, L. Warmerdam, F. Roozeboom, and H. Bergveld, "Voltage conversion circuit," Apr. 2011, EP Patent App. EP20,090,171,834. [Online]. Available: https://www.google.com/patents/EP2306626A1?cl=zh

[23] A. Sarafianos, J. Pichler, C. Sandner, and M. Steyaert, "A folding dickson-based fully integrated wide input range capacitive DC-DC converter achieving Vout/2-resolution and $71 \%$ average efficiency," in 2015 IEEE Asian Solid-State Circuits Conference (A-SSCC), Nov 2015, pp. 1-4.

[24] H. Meyvaert, T. V. Breussegem, and M. Steyaert, "A 1.65 W fully integrated $90 \mathrm{~nm}$ bulk cmos capacitive DC-DC converter with intrinsic charge recycling," IEEE Transactions on Power Electronics, vol. 28, no. 9, pp. 4327-4334, Sept 2013.

[25] J. Cools and P. Reynaert, "A 40nm bulk CMOS line driver for broadband communication," in ESSCIRC Conference 2016: 42nd European Solid-State Circuits Conference, Sept 2016, pp. 273 276.

[26] Y. Ismail, H. Lee, S. Pamarti, and C. K. K. Yang, "A 36-V 49\% Efficient Hybrid Charge Pump in Nanometer-Scale Bulk CMOS Technology," IEEE Journal of Solid-State Circuits, vol. 52, no. 3, pp. 781-798, March 2017.

[27] N. Butzen and M. Steyaert, "A 1.1W/mm2-Power-Density 82\%Efficiency Fully Integrated 3:1 Switched-Capacitor DC-DC Converter in Baseline 28nm CMOS using Stage Outphasing and Multiphase Soft-Charging," in 2017 IEEE International SolidState Circuits Conference (ISSCC), Feb 2017, pp. 178-179. 